\title{
Interstellar Scintillation and Intraday Variability
}

\author{
Barney J. Rickett \\ ECE Dept, University of California San Diego, La Jolla, CA92093-0407, USA
}

Abstract. Sources that are compact enough to show intrinsic variability on times of a day or less (IDV) at $\mathrm{cm}$ wavelengths must also show interstellar scintillation (ISS) on similar timescales. However for many IDV sources, the variations could be entirely due to ISS, reducing the implied brightness temperatures to $\sim 10^{13} \mathrm{~K}$ or less.

As discussed by Walker (these Proceedings, p. 285) and Rickett (1986), ISS can be an important cause of rapid flux variations at $\mathrm{cm}$ wavelengths for sufficiently compact sources. Sufficiently compact here means effective diameters $\lesssim 8\left(L_{\mathrm{kpc}} / f_{\mathrm{GHz}}\right)^{0.5} \mu$ as (Rickett 1990) for a path length $L_{\mathrm{kpc}}$ through the irregular plasma. At Galactic latitudes above $\sim 30^{\circ}$ this length can be $\lesssim 1 \mathrm{kpc}$, so that any AGN components smaller than about $100 \mu$ as must fluctuate randomly on times of an hour or longer. The exact timescale and rms amplitude depend on frequency and the compact source structure.

It should be noted, however, that the ISS interpretation of the IDV from quasar $0917+624$ (Rickett et al. 1995) required that the scattered path length be reduced from $1 \mathrm{kpc}$, as deduced from the Taylor \& Cordes (1993) model, to $200 \mathrm{pc}$. We then found good agreement with the observations by modeling the compact core as $0.6 \mathrm{Jy}$ in $70 \mu$ as at $6 \mathrm{~cm}$, i. e. a brightness temperature of $6.10^{12}$ $\mathrm{K}$-five orders of magnitude smaller than needed for the simplest intrinsic explanation. Alternative intrinsic models have invoked jets with extreme Doppler boosting factors $\sim 100$, compared with the much more normal value $\sim 2$ implied by the ISS interpretation. In their VLBI study Standke et al. (1996) identified $1.4 \mathrm{Jy}$ in a 0.2 mas component. Though 0.2 mas is too large to scintillate, their data appear to be consistent with $0.6 \mathrm{Jy}$ in an unresolved core.

The key ISS signature in the flux variations of $0917+624$ is the highly correlated nature of the fluctuations observed simultaneously at $2,3.6,6$, and 11 $\mathrm{cm}$, with a timescale increasing with wavelength. This behavior is exactly as predicted for ISS, partially quenched by smoothing over an angular diameter increasing linearly with wavelength. The scintillations at 3.6 and $11 \mathrm{~cm}$ are timealigned to better than $1 \mathrm{hr}$, from which we deduce a positional alignment to better than $10 \mu$ as for their centroids of emission. This puts a limit on the "core shift", in the (unknown) direction of the transverse velocity of the diffraction pattern relative to the earth. An independent confirmation of the ISS interpretation comes from observations of the scintillation of the pulsar PSR B0917+63 at $408 \mathrm{MHz}$, which probes a $700 \mathrm{pc}$ line of sight within half a degree of the quasar; its diffractive scintillation parameters are entirely consistent with the scattering deduced for $0917+624$ (Rickett \& Lyne, in preparation).

Heeschen et al. (1987) studied the flickering at a few \% of compact sources over a day or two at $\mathrm{cm}$ wavelengths. Quirrenbach et al. (1992), in their survey, concluded that most IDV sources formed a continuum with flickering sources and that their common origin was probably ISS. They also argued that $10-15 \%$ variations were intrinsic. Among such sources, $0917+624$ and $0716+714$ have been much studied (Wagner \& Witzel 1996; Wagner, these Proceedings p. 257). In light of the ISS interpretation for $0917+624$, one must ask whether all IDV could be attributed to ISS. There is no possibility that ISS could be responsible for any 
hour-by-hour correlation between radio and optical fluxes. In a comprehensive review of $0716+714$, Wagner et al. (1996) argue that the similarity in radio and optical flux variations in 1990 supports an intrinsic origin. Though the formal correlation coefficient is not high, there is a striking similarity in the character of the variations in this one well-studied epoch. One must then estimate the $6 \mathrm{~cm}$ ISS contribution, expected from a source small enough to vary intrinsically over 12 hours. Pulsar PSR B0809+74 lies 4 degrees away and probes half of the line of sight. Its scintillations at $30 \mathrm{~cm}$ scaled to $6 \mathrm{~cm}$ give an rms amplitude of about $15 \%$ over 5 hours (Rickett, Coles, \& Markkanen, in preparation), and the predicted amplitude and timescale for $0716+714$ are comparable to those observed. I am not here arguing that all of its $6 \mathrm{~cm}$ variations are ISS, but that any interpretation must include the ISS.

There are now several extragalactic sources with documented ISS contributions. The fastest IDV yet reported are from PKS 0405-385 are reported by Kedziora-Chudczer et al. (these Proceedings, p. 267). A crude estimate of the brightness temperature $T_{12}$ in unit of $10^{12} \mathrm{~K}$ needed for an ISS explanation is given by equation (6) of Rickett (1986).

$$
S_{\mathrm{rms}} / \tau \sim 30 T_{12}(\sin b)^{0.5} \mathrm{mJy} \mathrm{day}^{-1}
$$

The timescale $\tau=1 \mathrm{hr}$ and $\mathrm{rms}$ flux $S_{\mathrm{rms}}=500 \mathrm{mJy}$, at latitude $b=45^{\circ}$ giving a brightness $\sim 5.10^{14} \mathrm{~K}$. This is a hundred times that for $0917+624$ but still a million times below that implied by the simple intrinsic interpretation! The IDV from 0405-385 were observed over several months and then disappeared; this suggests that an extremely small component, causing ISS, was ejected from the core and, as it expanded, became too large to scintillate.

IDV in the linearly polarized flux and angle are sometimes also observed, partially correlated with the total flux. Whereas ISS cannot cause the rapid polarization variations of a single compact source, the combination of partially independent scintillations of polarized components each with fixed percent and angle of polarization can cause substantial rapid variations in the overall Stokes' parameters. Such models have been attempted for $0917+624$ (Rickett et al. 1995) with only partial success. Nevertheless, there is great potential for such analyses in the future (e.g., for 0405-385). Thus though ISS may appear to be a nuisance, it is exciting that its analysis can provide source structure information on scales finer than from Earth-based VLBI.

Acknowledgments. This work is supported by the US NSF under grant AST-94141444

\section{References}

Heeschen, D. S., et al. 1987. $A J$, 94, 1493-1507.

Quirrenbach, A., et al. 1992. A\&A, 258, 279-284.

Rickett. B. J. 1986. ApJ, 307, 564-574.

Rickett. B. J. 1990. ARA\&A, 28,561-605

Rickett. B. J., et al. 1995. $A \& A, 293,479-492$

Standke, K. J., et al. 1996. $A \mathscr{E} A$, 306, 27-38.

Taylor. J. H., \& Cordes, J. M. 1993. $A p J, 411,674-684$.

Wagner, S. J., \& Witzel, A. 1996. ARA\&A, 33, 163-197.

Wagner, S. J., et al. 1996. $A J, 111,2187-2211$. 